\title{
Opinión de las enfermeras de salud mental de España sobre el enfoque de recuperación
}

Opinion of the Spanish Mental Health Nurses About the Recovery Perspective

\section{José Luís Molino Contreras ${ }^{1}$ Evelyn Huizing ${ }^{2}$, Ana $\mathbf{M}^{\mathrm{a}}$ Pérez Vera ${ }^{3}$, José Antonio López Cócera ${ }^{3}$, Grupo Recuperación y Cuidados ${ }^{4}$}

\author{
'Facultad de Enfermería de Cartagena. Universidad de Murcia. Cartagena, España. \\ ${ }^{2}$ Servicio Andaluz de Salud. Sevilla, España. \\ ${ }^{3}$ Consejería de Salud Universal y Salud Pública de Valencia. Valencia, España. \\ ${ }^{4}$ Assumpta Rigol Cuadra, Encarnación Betolaza López de Gámiz, Leonor Padilla Obrero, Luna Serna Montero. \\ Contacto: josel.molino@carm.es \\ Fecha de recepción: 28 de junio de 2016 / Fecha de aceptación: 15 de septiembre de 2016
}

\begin{abstract}
Resumen
Objetivo: Conocer la opinión de las enfermeras españolas sobre la prestación de cuidados a pacientes con trastorno mental desde el enfoque de recuperación.

Metodología: Estudio descriptivo correlacional del año 2013. Población del estudio: conjunto de enfermeras que trabajan en los servicios públicos de salud mental de España. Se utilizó una combinación de muestreo por bola de nieve y por cuota, enviando un cuestionario online a 949 enfermeras. El tamaño muestral fue ponderado por número de habitantes en cada comunidad autónoma. Se utilizó el cuestionario Recovery Self Assessment-Registered Nurse (RSA-RN), traducido y adaptado al entorno español.

Resultados: Participaron enfermeras de todas las comunidades autónomas y de las dos ciudades autónomas. Se consiguieron un total de 503 cuestionarios (53\%). Se encontró una puntuación media de un 4,23 sobre 5 en el RSARN. No se encontró un perfil concreto de enfermeras con una opinión más positiva hacia la recuperación. La única variable que marcó una diferencia significativa fue tener formación como especialista vía enfermera interna residente, independientemente de la edad ( $p=0,002$ como valor resumen).

Conclusiones y discusión: Se encontró una actitud muy positiva hacia el enfoque de recuperación, con diferencia entre subescalas de la RSA-RN. Hay una necesidad de formación y sensibilización sobre la importancia de la participación activa de los usuarios en los servicios y cómo enfocar nuestro trabajo sobre las fortalezas del usuario, y cómo facilitar su participación real.

Enfermería comparte muchos de los valores del enfoque de recuperación y puede jugar un rol clave para impulsar la recuperación desde los servicios. Es necesario un apoyo institucional para fomentar el trabajo desde este enfoque y se debe avanzar en el desarrollo de intervenciones basadas en la recuperación. Asimismo, es fundamental monitorizar la implementación de la perspectiva de recuperación en los servicios.
\end{abstract}

Palabras clave: enfermería, salud mental, trastorno mental, recuperación, empoderamiento, España.

\begin{abstract}
Objective: Know the opinion of the Spanish mental health nurses about providing nursing care for patients with mental disorders from a recovery perspective.

Methodology: Descriptive correlational study carried out in the year 2013. Population of the study: nurses that work in the public mental health services of Spain. We used a combination of snowball and quota sampling, sending a questionnaire online to 949 nurses. The size sample was weighted according to the number of inhabitants in every autonomous community. The Recovery Self-Assessment-Registered Nurse (RSA-RN) questionnaire was used, after being translated and adapted to the Spanish environment.

Results: Nurses of all the autonomous communities and the two autonomous cities of Spain participated. We gathered 503 questionnaires (53\%). We found a scoring average of 4.23 out of 5 in the RSA-RN. A concrete profile of nurses with a more positive opinion towards recovery related to the socio-demographic variables wasn't found. The only variable that marked a significant difference was the training as a mental health nurse specialist via the Nurse Residency Program, regardless of age ( $p=0.002$ as summary score).
\end{abstract}


Conclusions and discussion: A very positive attitude towards the recovery approach was found, with difference between subscales of the RSA-RN. There is a need for training and awareness raising about the importance of active service user participation and on focusing on strengths of the service users, and facilitating their real participation. Nurses share many of the values of the recovery approach and can play a key role in promoting recovery within the services. Institutional support is necessary in order to foster the work from this approach and to progress in the development of interventions based on recovery. It is also essential to monitor the implementation of recovery in the services.

Keywords: nurses, mental health, mental disorders, recovery, empowerment, Spain.

\section{Introducción}

El término recuperación, recovery en inglés, es difícil de delimitar, ya que no existe una definición universal aceptada. En la literatura se aborda el concepto de recuperación como modelo, filosofía, paradigma, movimiento, visión o enfoque ${ }^{1}$. Sin embargo, sí existe un amplio consenso en que la definición estaría en torno a lo dicho por uno de los fundadores intelectuales del movimiento de la recuperación, William Anthony: "La recuperación es un proceso único, hondamente personal, de cambio de actitudes, valores, sentimientos, metas, habilidades y roles de una persona. Es una manera de vivir una vida satisfactoria, con esperanza y aportaciones, incluso con las limitaciones causadas por la enfermedad. La recuperación implica desarrollar un nuevo sentido y propósito en la vida, a la vez que la persona crece más allá de los efectos catastróficos de la enfermedad mental" ${ }^{2}$. Por tanto, una persona puede recuperar su vida (recuperación social) sin necesariamente recuperarse de su enfermedad (recuperación (línica) $)^{3,4}$.

Los servicios basados en la recuperación se apoyan en dos supuestos. Primero, que no todos los problemas mentales son crónicos y, segundo, que las personas con más probabilidades de recuperarse son las que tienen una motivación muy fuerte para cambiar sus vidas. Esta motivación está muchas veces condicionada por la actitud y el comportamiento de las personas de su entorno, cuando les apoyan en sus ambiciones personales y en la esperanza de tener una vida mejor ${ }^{5}$.

Para que la recuperación tenga el impacto que merece, los profesionales necesitan entender qué significa y, junto con pacientes y familiares, respaldar activamente su implementación en los servicios.

Pero los profesionales y los servicios de salud mental por sí solos no pueden hacer realidad la recuperación, ya que la llevan a cabo los propios usuarios y sus familias. Sin embargo, los profesionales pueden crear las condiciones en las cuales las personas se sientan con poder de decisión, para que la autogestión personal pueda desarrollarse, evitando en todo momento la creación de condiciones en las que la práctica de la recuperación resulte imposible ${ }^{3,4,6}$.

Diferentes estudios identificaron las características claves que deben tener los profesionales para trabajar desde la perspectiva de recuperación: mostrar una actitud de apertura, colaborar de igual a igual, centrarse en recursos internos de la persona, mostrar reciprocidad y tener la voluntad de ir más allá ${ }^{1,7}$.

Además, estas características generales deben estar combinadas con un alto nivel de habilidades relacionales: empatizar, atender-cuidar, aceptar, reconocimiento mutuo, animar a asumir riesgos responsables, tener actitudes positivas de cara al futuro y crear relaciones que inspiren esperanza. De cualquier modo, conseguir prácticas orientadas a la recuperación significaría conseguir un cambio en la cultura y en la organización de los servicios ${ }^{1,7}$.

La perspectiva de recuperación ha sido ampliamente aceptada por las enfermeras, por representar valores y competencias muy ligados a los cuidados, como la relación entre enfermera y paciente centrada en la persona, el enfoque holístico, el uso de la narrativa y el dialogo más natural, las relaciones basadas en la empatía y el calor humano, la promoción de la salud y la relación con personas usuarias en contextos varios, dentro y fuera de los servicios ${ }^{8-22}$.

El objetivo de este estudio es conocer la opinión de las enfermeras españolas sobre la prestación de cuidados a pacientes con trastorno mental desde el enfoque de recuperación, según las cinco subescalas del RSARegistered Nurse versión (RSA-RN) ${ }^{23}$, y en relación a las variables sociodemográficas de las enfermeras identificadas como relevantes. 


\section{Metodología}

Es un estudio descriptivo correlacional desarrollado en el año 2013. La población del estudio fue el conjunto de enfermeras que trabajan en el área de salud mental de los servicios públicos de las 17 comunidades autónomas (CCAA) y las ciudades autónomas de Ceuta y Melilla. Se utilizó una combinación de muestreo por bola de nieve y por cuota, enviando un cuestionario online a 949 enfermeras, de las aproximadamente 4000 enfermeras de salud mental estimada por la Asociación Nacional de Enfermería de Salud Mental (ANESM). El tamaño muestral fue ponderado por el porcentaje que representan los habitantes de cada comunidad autónoma (CA) en el conjunto de España según los datos del Instituto Nacional de Estadística $2011^{24}$.

Se identificaron 19 enfermeras (17 CCAA y 2 ciudades autónomas de Ceuta y Melilla), a través del listado de socios enfermeras de la ANESM, que a su vez identificaron a un número de enfermeras ponderado por CA, que posteriormente enviaron a 10 enfermeras el cuestionario en la siguiente proporción: asistencial $(80 \%)$, gestión $(10 \%)$, docencia $(10 \%)$. Así, por ejemplo, a la CA de Asturias le correspondían 30 cuestionarios, por lo que el referente de la ANESM identificó a 3 enfermeras que a su vez mandaron el cuestionario a diez enfermeras, ocho del ámbito asistencial, una del ámbito de gestión y una del ámbito de docencia.

Se incluyeron enfermeras generalistas y enfermeras especialistas en salud mental que llevaban al menos un año trabajando en los servicios de salud mental.

Quedaban excluidas las enfermeras con experiencia en salud mental y/o con el título de especialista en Salud Mental que no estaban trabajando en salud mental en el momento de la recogida de datos, así como las enfermeras internas residentes (EIR) de salud mental en periodo de formación.

Se definieron las siguientes variables sociodemográficas: edad, sexo, modalidad de acceso al título de enfermera especialista en Salud Mental, otra formación postgrado, años de experiencia laboral, trayectoria laboral en salud mental, conocimiento previo y/o formación en recuperación, y formación en rehabilitación psicosocial.

Para conocer la opinión sobre la perspectiva de recuperación se utilizó el cuestionario Recovery Self Assessment-Registered Nurse (RSA-RN) ${ }^{23}$, un instru- mento de 36 preguntas que traduce los principios de recuperación en medidas prácticas, permitiéndole detectar las fortalezas y las áreas de mejora. Las preguntas pertenecen a 5 factores o subescalas: "Objetivos de vida", "Participación activa de usuarios", "Diversidad en opciones de tratamiento", "Elección"y "Servicios personalizados". Las preguntas están aleatoriamente ordenadas y se presentan como un listado de 36 preguntas, sin que se sepa a qué subescala pertenece cada pregunta.

El RSA-RN es una de las versiones del Recovery Self Assesment (RSA) ${ }^{25}$, un cuestionario desarrollado por la Universidad de Yale, basado en los 9 dominios de la recuperación ${ }^{26}$, diseñado para valorar el grado en que los servicios han implementado prácticas basadas en la recuperación. Este instrumento tiene diferentes versiones: para proveedores (los profesionales asistenciales), para gestores (los directivos de los centros), para pacientes y para familiares. Para evaluar el grado de implementación de la perspectiva de recuperación en un servicio u organización se deben usar todas las escalas a la vez, ya que cada versión tiene su propia perspectiva y en su conjunto son complementarios.

El RSA de los proveedores es para todas las categorías profesionales y tipo de unidades, pero se entiende que va más dirigido a los servicios comunitarios. Por este motivo, se desarrolló en el 2008 una versión de proveedores para enfermeras diplomadas, la RSA$\mathrm{RN}^{23}$, en la cual adaptaron las preguntas al entorno hospitalario, donde trabaja un porcentaje más alto de enfermeras de salud mental en comparación con el entorno comunitario.

El cuestionario tiene una puntuación en una escala Likert de "muy en desacuerdo" [1] a "muy de acuerdo" [5], donde una puntuación más alta indica que el servicio se ajusta más a la los principios de recuperación. El objetivo fue encontrar la correlación de la opinión de la enfermera con sus variables sociodemográficas. La mayoría de estas variables fueron utilizadas en la construcción y validación del RSA-RN ${ }^{23}$.

Se tradujo y se adaptó el RSA-RN al entorno español y se modificaron las preguntas a una formulación condicional, utilizando el término "debería". El motivo de este cambio fue debido a que se pretendía conocer el grado de acuerdo en relación a que los ítems reflejaran las actividades, valores y prácticas de su servicio, y no en qué grado estaba la perspectiva de recuperación implantada en el servicio. 
La información fue recogida en un cuestionario online en el entorno Google Drive, un servicio de alojamiento de archivos.

Para conseguir una precisión del $3 \%$ en la estimación de una proporción mediante un intervalo de confianza asintótico normal con corrección para poblaciones finitas al $95 \%$ bilateral, se calculó que sería necesaria una muestra de 584 .

Se realizó estadística descriptiva de las variables del estudio. Para ello se usaron frecuencias absolutas y porcentajes en el caso de las variables cualitativas. Las variables cuantitativas fueron resumidas mediante media \pm SD. Se comprobó si las variables seguían una distribución normal mediante el test de Kolmogrov-
Smirnov. Se realizó una comprobación de los grupos de estudios mediante test $\chi^{2}$ o el test exacto de Fisher, cuando fue necesario para las variables cualitativas. $\mathrm{La}$ comparación de las variables cuantitativas según los grupos de estudio se efectuó mediante la t-Student para muestras independientes. El nivel de significación estadística se estableció en $p<0,05$. El análisis estadístico se realizó con el paquete Statistical Package for Social Sciences (SPSS Inc., Chicago, IL, EE. UU.), versión 19.0.

\section{Limitaciones}

Las limitaciones de los datos radican en el muestreo no probabilístico al producir estimadores sesgados, ya que personas muy populares dentro de la población

Tabla 1: Perfil de las enfermeras

\begin{tabular}{|c|c|c|c|}
\hline \multirow[t]{2}{*}{ Sexo } & \multicolumn{2}{|l|}{ Mujeres } & $74,4 \%$ \\
\hline & \multicolumn{2}{|c|}{ Hombres } & $25,6 \%$ \\
\hline Edad media & \multicolumn{3}{|c|}{44 años [rango 27-62] } \\
\hline \multirow[t]{3}{*}{ Fecha titulación enfermera diplomada } & \multicolumn{2}{|c|}{$1970-1979$} & $11,1 \%$ \\
\hline & \multicolumn{2}{|c|}{ 1980-1999 } & $66,4 \%$ \\
\hline & \multicolumn{2}{|c|}{$2000-2011$} & $22,5 \%$ \\
\hline \multirow[t]{4}{*}{ Especialidad en salud mental } & \multicolumn{2}{|c|}{ Anterior 1998} & $13,3 \%$ \\
\hline & \multicolumn{2}{|l|}{ Vía EIR } & $20,3 \%$ \\
\hline & \multicolumn{2}{|c|}{ Acceso extraordinario } & $42,1 \%$ \\
\hline & \multicolumn{2}{|c|}{ No especialidad } & $24,3 \%$ \\
\hline \multirow[t]{3}{*}{ Formación postgrado $>60$ créditos } & \multirow{3}{*}{$49,30 \%$} & Máster & $30,2 \%$ \\
\hline & & Lic. Psic./Antrop. & $10,7 \%$ \\
\hline & & Otra & $8,4 \%$ \\
\hline \multirow[t]{5}{*}{ Ámbito laboral actual } & \multicolumn{2}{|c|}{ Hospitalización } & $47,1 \%$ \\
\hline & \multicolumn{2}{|c|}{ Unidad/Programa Comunitario } & $33,8 \%$ \\
\hline & \multicolumn{2}{|c|}{ Gestión } & $14,1 \%$ \\
\hline & \multicolumn{2}{|c|}{ Docencia } & $4 \%$ \\
\hline & \multicolumn{2}{|l|}{ Otros } & $1 \%$ \\
\hline \multirow[t]{4}{*}{ Trabajo previo estable en salud mental } & \multicolumn{2}{|c|}{ Hospitalización } & $49,1 \%$ \\
\hline & \multicolumn{2}{|c|}{ Unidad/Programa Comunitario } & $19,2 \%$ \\
\hline & \multicolumn{2}{|c|}{ Ambas unidades } & $10,6 \%$ \\
\hline & \multicolumn{2}{|c|}{ Sin experiencia anterior } & $21,1 \%$ \\
\hline \multirow[t]{5}{*}{ Experiencia en salud mental } & \multicolumn{2}{|c|}{1 a 2 años } & $9,3 \%$ \\
\hline & \multicolumn{2}{|c|}{3 a 5 años } & $17,4 \%$ \\
\hline & \multicolumn{2}{|c|}{6 a 10 años } & $19,6 \%$ \\
\hline & \multicolumn{2}{|c|}{11 a 20 años } & $25,3 \%$ \\
\hline & $>20$ año & & $28,4 \%$ \\
\hline Conocimiento enfoque recuperación & Sí & & $50,5 \%$ \\
\hline & No & & $49,5 \%$ \\
\hline Formación recuperación & Sí & & $21,1 \%$ \\
\hline & No & & $78,9 \%$ \\
\hline Formación rehabilitación psicosocial & Sí & & $63,6 \%$ \\
\hline & No & & $36,4 \%$ \\
\hline
\end{tabular}


tuvieron mayores oportunidades de ser seleccionadas, lo que impide extrapolar los resultados a la población sin más.

\section{Resultados}

En el estudio participaron enfermeras de todas las CAy de las dos ciudades autónomas. Se ha obtenido una tasa de repuesta del $53 \%$ (503). El perfil de las enfermeras participantes se observa en la tabla 1 .

En cuanto al conjunto de las subescalas del RSA$\mathrm{RN}$, encontramos que la puntuación media es de un 4,23 sobre 5. Sin embargo, hay una diferencia entre las cinco subescalas. Las subescalas de menor puntuación son las de "Participación activa de los usuarios" y "Diversidad en opciones de tratamiento". La subescala "Elección" ha obtenido la mayor puntuación, seguida por "Objetivos de vida" y "Servicios personalizados". Ver tabla 2.

En cuanto a las cinco preguntas con menos puntuación en el conjunto del cuestionario, hay preguntas de tres subescalas. Con respecto a las cinco preguntas con más puntuación, igualmente hay preguntas de tres subescalas. Ver tabla 3.

Se valoró una puntuación de $<3$ como "en desacuerdo" y de $>3$ como "de acuerdo", excluyendo la puntuación $=3$ al no haber un posicionamiento a favor o en contra.

No se encontró un perfil concreto de enfermeras con una opinión más positiva hacia la recuperación, en relación a las variables sociodemográficas.

No hay una diferencia significativa en tener o no la especialidad en salud mental y tampoco entre sexos y años de experiencia en salud mental. No hay diferencia por edad (grupo $>38$ y $<38$ años) y tampoco entre tener un máster o no. Tampoco hay diferencia entre las que han recibido formación en recuperación y/o rehabilitación psicosocial y las que no, ni en cuanto al lugar actual de trabajo.

$\mathrm{Y}$ tampoco hay diferencia en las enfermeras con "Formación postgrado $>60$ créditos", salvo en la subescala "Participación activa del usuario", con una diferencia significativa en la unión del grupo de licenciados en Psicología/Antropología con el grupo de doctorado en comparación con el resto con formación postgrado $>60$ créditos.

La única variable que marca una diferencia significativa es tener formación vía EIR, independientemente de la edad. Hay una puntuación mayor en todas las subescalas. Ver tabla 4.

\section{Discusión}

En general, hay una actitud muy positiva por parte de las enfermeras de los servicios públicos de salud mental de España hacia el enfoque de recuperación, pero hay una necesidad de formación y sensibilización sobre la importancia de la participación activa de los usuarios en los servicios. También hay una necesidad de formación y sensibilización acerca de cómo enfocar nuestro trabajo en las fortalezas del usuario y ayudarle a explorar otros roles (servicios de "salud" mental vs. servicios de "enfermedad" mental), y de cómo facilitar la participación real de los usuarios en las instituciones, poniendo en valor el apoyo mutuo.

Los resultados de las cinco preguntas con más y menos puntuación coinciden en gran parte con otros estudios con el RSA en los Estados Unidos (EE. UU.), donde se ha utilizado la escala RSA en situación real de evaluación de servicios ${ }^{24,25,27}$. Esto es interesante, ya que estos parecen ser los ámbitos dónde más hace falta sensibilizar y profundizar, porque incluso cuando hay una política clara a favor de la implantación del enfoque de recuperación, son estas las áreas donde menos

Tabla 2: Puntuación media por subescala RSA-RN

\begin{tabular}{|l|l|}
\hline Subescala RSA-RN & Puntuación media \\
\hline Objetivos de vida (11 ítems) & 4,31 \\
\hline Participación activa de usuarios (8 ítems) & 4,04 \\
\hline Diversidad en opciones de tratamiento (6 ítems) & 4,15 \\
\hline Elección (6 ítems) & 4,45 \\
\hline Servicios personalizados (5 ítems) & 4,23 \\
\hline
\end{tabular}


Tabla 3: Preguntas con menor y mayor puntuación

\begin{tabular}{|c|c|c|}
\hline \multicolumn{3}{|c|}{ Las 5 preguntas con menos puntuación } \\
\hline Subescala & Pregunta & $\begin{array}{l}\text { Puntuación }<3 \\
{[\text { en desacuerdo] }}\end{array}$ \\
\hline \multirow[t]{3}{*}{$\begin{array}{l}\text { Participación activa de } \\
\text { usuarios }\end{array}$} & $\begin{array}{l}\text { Las personas en proceso de recuperación deberían participar } \\
\text { habitualmente en los órganos de gestión y reuniones de } \\
\text { enfermería. }\end{array}$ & $24 \%$ \\
\hline & $\begin{array}{l}\text { Las personas en proceso de recuperación deberían participar en } \\
\text { la formación de los profesionales de enfermería y en los } \\
\text { programas educativos del servicio. }\end{array}$ & $12 \%$ \\
\hline & $\begin{array}{l}\text { Las personas en proceso de recuperación deberían trabajar } \\
\text { conjuntamente con el equipo de enfermería en el desarrollo y } \\
\text { prestación de nuevos programas y servicios. }\end{array}$ & $7 \%$ \\
\hline $\begin{array}{l}\text { Diversidad de opciones } \\
\text { de tratamiento }\end{array}$ & $\begin{array}{l}\text { En el servicio, los usuarios que progresan bien deberían recibir la } \\
\text { misma cantidad de atención del equipo de enfermería que los que } \\
\text { tienen dificultades. }\end{array}$ & $19 \%$ \\
\hline Objetivos de vida & $\begin{array}{l}\text { El equipo de profesionales de enfermería debería ser diverso en } \\
\text { cuanto a cultura, etnia, estilos de vida e intereses. }\end{array}$ & $10 \%$ \\
\hline \multicolumn{3}{|c|}{ Las 5 preguntas con más puntuación } \\
\hline Subescala & Pregunta & $\begin{array}{l}\text { Puntuación }>3 \\
{[\text { de acuerdo }]}\end{array}$ \\
\hline \multirow[t]{3}{*}{ Elección } & $\begin{array}{l}\text { Las enfermeras y técnicos en cuidados auxiliares de enfermería de } \\
\text { este servicio deberían escuchar y atender las decisiones y } \\
\text { preferencias de los pacientes/usuarios. }\end{array}$ & $100 \%$ \\
\hline & $\begin{array}{l}\text { El progreso hacia los objetivos (definidos por el usuario en } \\
\text { proceso de recuperación) se debería de monitorizar de forma } \\
\text { habitual por parte de la enfermera referente / del equipo de } \\
\text { enfermería. }\end{array}$ & $99 \%$ \\
\hline & $\begin{array}{l}\text { Las enfermeras y técnicos en cuidados auxiliares de enfermería no } \\
\text { deberían usar amenazas, sobornos otras formas coercitivas para } \\
\text { influir en la conducta y elecciones del usuario. }\end{array}$ & $98 \%$ \\
\hline Objetivos de vida & $\begin{array}{l}\text { Deberían existir procedimientos para facilitar la derivación a otros } \\
\text { programas, si el equipo de enfermería siente que el servicio no } \\
\text { puede cubrir las necesidades del usuario. }\end{array}$ & $100 \%$ \\
\hline Servicios pers onalizados & $\begin{array}{l}\text { Se debería hacer todo lo posible por implicar a las personas } \\
\text { significativas del usuario (cónyuges, amigos, familiares) y otros } \\
\text { apoyos naturales (p. ej. la parroquia, vecinos, caseros) en el plan } \\
\text { de cuidados individualizado, si la persona lo desea. }\end{array}$ & $98 \%$ \\
\hline
\end{tabular}

Tabla 4: Nivel de significación estadística en las EIR

\begin{tabular}{|c|c|c|c|}
\hline \multirow[t]{2}{*}{ Subes cala RSA-RN } & No EIR & EIR & \\
\hline & $(n=401)$ & $(n=102)$ & $\mathbf{p}$ \\
\hline Objetivos de vida & $4,29(0,55)$ & $4,41(0,53)$ & 0,039 \\
\hline Participación activa de usuarios & $3,99(0,68)$ & $4,25(0,60)$ & 0,000 \\
\hline Diversidad en opciones de tratamiento & $4,12(0,62)$ & $4,28(0,57)$ & 0,014 \\
\hline Elección & $4,43(0,51)$ & $4,59(0,44)$ & 0,002 \\
\hline Servicios pers onalizados & $4,20(0,63)$ & $4,40(0,50)$ & 0,001 \\
\hline Valor resumen & $4,20(0,53)$ & $4,38(0,48)$ & 0,002 \\
\hline
\end{tabular}


se ha avanzado. De todos modos, la pregunta sobre la diversidad de los equipos de enfermería en cuanto a cultura, etnia, etc., es más relevante en los EE. UU. al ser una sociedad mucho más multirracial y multicultural que España y puede que las enfermeras españolas no hayan dado tanta importancia a esta pregunta por este motivo.

En cuanto a los resultados de las EIR, pensamos que esta puntuación es más alta por la visión más amplia que tienen de la red de salud mental debido a la rotación en los servicios, y por tener otro rol en los equipos de trabajo al estar en formación postgrado. Las EIR disponen de más tiempo y espacio de reflexión fuera del servicio y del trabajo diario, tanto entre ellas como con los docentes de la especialidad y con los otros compañeros de la Unidad Docente Multiprofesional (Psiquiatría y Psicología Clínica).

De todos modos es importante recordar que en este estudio se ha medido la opinión de las enfermeras españolas sobre la prestación de cuidados a pacientes con trastorno mental desde el enfoque de recuperación, usando la formulación condicional "debería”, y no el grado en que están trabajando en la actualidad desde el enfoque de recuperación.

Los principios de la recuperación nos brindan una gran oportunidad para mejorar la práctica clínica en salud mental y enfermería comparte muchos de los valores de esta perspectiva ${ }^{8,18}$, pudiendo jugar un rol clave para impulsar la recuperación desde los servicios. Es necesario que haya un apoyo institucional para fomentar el trabajo desde este enfoque ${ }^{3,4,28}$, a través de formación en recuperación, traducción y distribución de documentos en castellano para facilitar el acceso a la información, colaboración estrecha con los movimientos asociativos de usuarios y familiares ${ }^{29}$, debate en los equipos de trabajo sobre los conceptos de la recuperación, etc. Se debe avanzar en el desarrollo de intervenciones basadas en la recuperación centradas en tres temas centrales: la esperanza, los cuidados centrados en la persona y tener en cuenta la perspectiva de los usuarios $^{30}$.

Asimismo, es fundamental monitorizar la implementación de la perspectiva de recuperación en los servicios ${ }^{29,31}$ con instrumentos validados, porque incluso en organizaciones donde hay una apuesta clara por trabajar desde el enfoque de recuperación, hay margen de mejora en todos los dominios de recuperación ${ }^{24,25,29}$.

\section{Conclusiones}

Una vez se avanzara en la implementación del enfoque de recuperación, sería interesante medir en qué grado está la perspectiva de recuperación implantada en los servicios a través de las diferentes versiones del RSA.

Para conseguir la implementación de la perspectiva de recuperación en los servicios es importante que enfermería se empodere dentro de la organización para así facilitar el empoderamiento de los usuarios y sus familiares $^{31}$, con el objetivo de mejorar los resultados en salud. Estar empoderado como colectivo profesional significa tener una sensación de competencia, de autonomía, de trabajo significativo y de capacidad para tener impacto en la organización. Para este empoderamiento, y desde la perspectiva de la recuperación, es fundamental centrarnos en los valores y principios de la propia disciplina enfermera y trabajar en la promoción de la salud, en fomentar el autocuidado, en facilitar el ejercicio de los derechos humanos, en el entorno natural del paciente, desde la relación terapéutica y la presencia.

\section{Agradecimientos}

A Juan Manuel Praena Fernández y Luís López Rodríguez, de la Unidad de Investigación del Hospital Universitario Virgen del Rocío de Sevilla, por su análisis estadístico y apoyo metodológico. A los referentes de las comunidades autónomas por la coordinación y a las enfermeras por su participación en el estudio.

\section{Bibliografía}

1. Cleary A, Dowling M. Knowledge and attitudes of mental health professionals in Ireland to the concept of recovery in mental health: a questionnaire survey. J Psychiatr Ment Health Nurs. 2009; $16: 539-545$

2. Anthony W.A. Recovery from mental illness: the guiding vision of the mental health service system in the 1990s. Psychiatr Rehabil J. 1993; 16: $11-23$.

3. Shepherd G, Boardman J, Slade M. Making Recovery a Reality Sainsbury Centre for Mental Health 2008.

4. Shepherd G, Boardman J, Burns M. Implementing recovery. A methodology for organisational change. Sainsbury Centre for Mental Health 2010

5. Mental Health Providers Forum. Recovery Star Approach. Disponible en: http://www.mhpf.org.uk/programmes-and-projects/mentalhealth-and-recovery/recovery-star [Consultado 22 de noviembre 2011].

6. Kartalova-O'Doherty Y, Tedstone Doherty D. Recovering from recurrent mental health problems: Giving up and fighting to get better. Int J Ment Health Nurs. 2010; 19: 3-15.

7. Lakeman R. Mental health recovery competencies for mental health workers: A Delphi study. JMH. 2010;19(1):62-74.

8. Caldwell B A, Sclafani M, Swarbrick M, Piren K. Psychiatric nursing 
practice \& de recovery model of care. J Psychosoc Nurs Ment Health Serv. 2010; Vol. 48: No. 7.

9. Shanley E, Jubb-Shanley M. The recovery alliance theory of mental health nursing. J Psychosoc Nurs Ment Health Serv. 2007; 14: $734-743$.

10. Jubb-Shanley M, Shanley E. Trialling of the Partnership in Coping system. J Psychosoc Nurs Ment Health Serv. 2007; 14: 226-232

11. Happell B. Lead by vision, not by limitations: Recovery and the mental health nursing profession. Int J Ment Health Nurs. 2010; 19:1-2.

12. Barker P. The Tidal Model: developing an empowering, personcentred approach to recovery within psychiatric and mental health nursing. J Psychosoc Nurs Ment Health Serv. 2001; 8:233240.

13. Barker P, Buchanan-Barker P. The Tidal Model of Mental Health Recovery and Reclamation: Application in Acute Care Settings. Issues Ment Health Nurs. 2010 Mar; 31:171-180.

14. Buchanan-Barker P, Barker P.J. The Tidal Commitments: extending the value base of mental health recovery. J Psychiatr Ment Health Nurs. 2008; 15, 93-100.

15. Cook NR, Philips BN, Sadler D. The tidal model as experienced by patients and nurses in a regional forensic unit. J Psychiatr Ment Health Nurs. 2005; 12: 536-540.

16. Brookes N, Murata L, Tansey M. Guiding practice development using the Tidal Commitments. J Psychiatr Ment Health Nurs. 2006; 13: $460-463$.

17. Barker P. The Tidal model. J Psychosoc Nurs Ment Health Serv. Jul 2002; 40 (7): 42-50.

18. Roe D, Swarbrick M. A recovery-oriented approach to psychiatric medication: Guidelines for nurses. J Psychosoc Nurs Ment Health Serv. 2007; 45(2), 35-40.

19. Happell B. Lead by vision, not by limitations: Recovery and the mental health nursing profession. Int J Ment Health Nurs. 2010; 19:1-2.

20. Health Department. Rights, relationship and recovery. The report of the National Review of Mental Health Nursing in Scotland. Main Report. Scottish executive. NHS Scotland 2006. Disponible en: http://www.scotland.gov.uk/Resource/Doc/112046/0027278.p df [Consultado 23 agosto 2016].

21. Health Department. Rights, relationship and recovery. The report of the National Review of Mental Health Nursing in Scotland. Delivery action plan. Scottish executive. NHS Scotland 2006.

22. From values to action: The Chief Nursing Officer's review of mental health nursing. 2006.

23. Mcloughlin KA, Fitzpatrick JJ. Self-reports of recovery-orientated practices of mental health nurses in state mental health institutes: development of a measure. Issues Ment. Health Nurs. 2008; 29:1051-1065.

24. Instituto Nacional de Estadística (INE). Disponible en: http://www.ine.es/censos2011_datos/cen11_datos_inicio.htm. [Consultado: 16 de octubre 2016].

25.0'Connel M, Tondora J,Croog G, Evan A, Davidson L. From rhetoric to routine: Assessing perceptions of recovery-orientated practices in a state mental health and addiction system. Psychiatr Rehabil J. 2005; 2884:378-386.

26. Davidson L, O'Connel M., Sells,D,Staeheli M. Is there an outside to mental illness? In L. Davidson. Living Outside Mental Illness. Qualitative Studies of Recovery in Schizophrenia. New York University Press, New York 2003;31-60.

27. McLoughlin KA, Du WickA, Collazzi CM, Puntil C. Recovery-Oriented practices of psychiatric-mental health nursing staff in an acute hospital setting. J Am Psychiatr Nurses Assoc. 2013; 19(3)-152159.

28. Gilburt et al. Promoting recovery-oriented practice in mental health services: a quasi-experimental mixed-methods study. BMC Psychiatry. 2013; 13:167. Disponible en: http://www.biomedcentral.com/1471-244X/13/167 [Consultado: 24 de agosto 2016 ].
29. Gomez Beneyto M. Introducción: En primera persona. Rev Asoc.Esp. Neuropsiq. 2016; 36(129):191-192.

30. Nursing Practice Review Mental Health. Recovery-focused practice in mental health. Nursing Times. 19.03.14. Vol110. No 12. 2014

31. User empowerment in mental health. A statement by the WHO Regional Office for Europe 2010. 